\title{
Aprendizaje Basado en Proyectos Reales Aplicado a la Formación del Ingeniero de Software
}

\author{
Marco A. Villalobos-Abarca ${ }^{(1) \star}$, Raúl A. Herrera-Acuña ${ }^{(1)}$, Ibar G. Ramírez ${ }^{(1)}$ y Ximena C. Cruz ${ }^{(2)}$ \\ (1) Universidad de Tarapacá, Esc. Univ. de Ing. Indus., Inf. y Sis., Especialidad de Ingeniería en \\ Computación e Informática, Casilla 7-D, Arica-Chile \\ (2) Universidad de Tarapacá, Fac. de Educación y Humanidades, Depto. Educación, Casilla 7-D, Arica-Chile
}

* Autor a quien debe ser dirigida la correspondencia

Recibido Sep. 15, 2017; Aceptado Nov. 24, 2017; Versión final Dic. 26, 2017, Publicado Jun. 2018

\begin{abstract}
Resumen
Las reflexiones sobre cómo dar un contexto más profesional al aprendizaje del desarrollo de software en la carrera de Ingeniería en Computación e informática, condujeron a que, en la Universidad de Tarapacá de Arica, Chile, se haya implementado una estrategia pedagógica que acercó al estudiante hacia problemas reales en contextos reales. Posteriormente, se indagó su impacto en el ejercicio temprano de la profesión de los egresados de la carrera mencionada. Se encuestó a exalumnos que desarrollaron proyectos reales en empresas reales y se entrevistó a los docentes, empresarios y un experto. Los resultados, permiten afirmar que los egresados dan una alta valoración a la estrategia y a sus características, incluso dando recomendaciones para mantener o aumentar sus énfasis. Así mismo, tanto los empresarios como los docentes, opinan que la experiencia es positiva y aporta hacia una formación más real y contextualizada en la realidad profesional en los alumnos.
\end{abstract}

Palabras clave: ingeniería de software; ABP; proyectos reales; PYMES; ejercicio temprano de la profesión

\section{Real Project-Based Learning Applied to Software Engineers' education}

\begin{abstract}
The reflections about how to give a more professional context to the software development on the degree of Informatics' Engineering led to the implementation of a pedagogic strategy at the Universidad de Tarapacá, Chile, to confront students with real problems under real contexts. Afterwards the impact of an early involvement on professional exercise by graduated students of the previously mentioned degree was enquired. Alumni that developed projects in real business were surveyed and professors, business owners and an expert were interviewed. The results obtained allow establishing that graduated students highly value this strategy and its characteristics, even recommending keeping or increasing the emphasis on the use of the strategy. In the same way, employers and lecturers as well address the positive impact of the strategy and how it helps to develop a more realistic professional experience for students.
\end{abstract}

Keywords: software engineering; PBL; real projects; PYMES; early exercise of professional skills 


\section{INTRODUCCIÓN}

Existe una brecha marcada entre lo que se enseña en el aula de clase y la realidad del desarrollo de software en las organizaciones. Esta situación ha motivado una reflexión en el mundo académico acerca de las competencias y habilidades que deben ser desplegadas por los futuros desarrolladores de software y las estrategias pedagógicas que pueden ser utilizadas de manera que sus experiencias de aprendizaje estén altamente influenciadas por las prácticas, técnicas y modos de trabajo que exige el desarrollo de software de calidad a escala industrial (Stiller y LeBland, 2002; Liu et. al., 2002; Bracken, 2003; Alsmadi y Abul-Huda, 2010). Por lo anterior, en la Universidad de Tarapacá de Arica, Chile (UTA) se ha implementado una estrategia pedagógica que acerca al estudiante hacia problemas reales en contextos reales. Es decir, el desarrollo de proyectos que resuelven problemas de micro empresas.

Actualmente el $\mathrm{ABP}$ ha tenido una gran aceptación en la educación superior, sin embargo la abreviación ABP se ha utilizado tanto para el aprendizaje basado en problema como para el aprendizaje basado en proyecto. Kolmos et al. (2009), establecen que hay razones para unificarlos a nivel de principios del aprendizaje. El aprendizaje basado en problema si bien surge como estrategia didáctica, más claramente en escuelas de medicina, se ha aplicado en otros ámbitos educativos dando lugar a múltiples modelos. Por su parte, Graaff y Kolmos (2007) señalan, aunque hay diferencia a nivel de modelos, que existen principios de aprendizaje comunes en los modelos de ABP y que corresponden a tres aproximaciones al aprendizaje: cognitivo, contenidos y colaborativo. En la tabla 1, se describen estos principios.

Así, se han propuesto diferentes modelos de ABP, en ellos se incluyen sus elementos básicos: conocimientos, aprendizajes, escenarios del problema, estudiantes, rol del profesor y evaluación. Kolmos, Graaff y Du (2009), presentan los cinco modelos de ABP, basado en los estudios de Sabin-Banden (2000) y Sabin-Banden (2007), entre los que se tienen: (I) Aprendizaje basado en problemas para la competencia, (II) Aprendizaje basado en problemas para la acción profesional, (III) Aprendizaje basado en problemas para la comprensión interdisciplinaria, (IV) Aprendizaje basado en problemas para el aprendizaje transdisciplinario y (V) Aprendizaje basado en problemas para desarrollar una actitud crítica. De los cinco modelos indicados, el que posee características más cercanas al trabajo realizado es el modelo II ya que por ejemplo, está orientado al aprendizaje en el lugar de trabajo o el escenario de los problemas a resolver, ocurren en la vida real. En la tabla 2, se muestran los elementos de dicho modelo.

En la formación del ingeniero informático de la UTA, los proyectos se transformaron en el eje de la acción formativa y aparecen como una estrategia importante, pues los estudiantes: (1) deben hacer frente a información incompleta o imprecisa, (2) deben autorregularse y comprometerse con el trabajo, involucrándolos en el proceso de aprendizaje, ya que deben definir sus propios objetivos dentro de los límites impuestos, (3) deben cooperar y trabajar en grupos dividiendo la carga de trabajo entre sí e integrar las diferentes partes desarrolladas por ellos y (4) trabajar con temas multidisciplinarios (problemas complejos implican varias disciplinas). Estas habilidades prácticas son un requisito importante de las empresas y la industria, como lo señalan Macías-Guarasa et al. (2006) y Reeves et al. (2002).

Por lo anterior, el ABP es relevante para el trabajo desarrollado, ya que es un método de enseñanza sistemático en que participan los estudiantes en aprendizajes de conocimientos esenciales y habilidades para la vida a través de un extendido y estructurado proceso de indagación influido por el estudiante alrededor de complejas preguntas auténticas y creando productos de alta calidad. También plantean que el $\mathrm{ABP}$ es un enfoque de instrucción construido sobre actividades auténticas o reales. En la tabla 3 , se describen las características de las actividades auténticas de aprendizajes que se logran con el ABP. Por lo tanto, no basta con aprender cuestiones técnicas y otros temas; los conocimientos técnicos deben ser utilizados en situaciones reales. Es importante destacar las conexiones entre diferentes aspectos, para tener una visión amplia de los sistemas y para ilustrar las restricciones prácticas, tecnológicas y las limitaciones humanas de la resolución de problemas en el mundo real.

Así considerando todo lo anterior y dado que uno de los requerimientos de la formación de ingenieros, en particular los informáticos "es que los estudiantes resuelvan problemas reales en contextos reales", en un curso denominado "Taller de Proyectos de Software" de la citada Universidad, se propuso que el escenario de los problemas sean las pequeñas empresas del entorno inmediato (Arica, Chile), que el aprendizaje fuera direccionado al desarrollo de competencias y que la evaluación fuera respecto del desempeño en el trabajo efectivo. El rol del profesor, en este contexto, fuera el acompañar al estudiante para que este pueda demostrar las habilidades prácticas requeridas. Por último, se tomó en consideración las características de las actividades auténticas descritas por Reeves et al. (2002) para evaluar si estas se encuentran presentes en los proyectos que ellos realizan y cuál es la valoración que ellos le asignan a cada una. 
Tabla 1. Principios de aprendizaje del ABP (Adaptado de Kolmos, (2009))

\begin{tabular}{|c|c|c|}
\hline Aprendizaje Cognitivo & Contenidos del Aprendizaje & Aprendizaje Colaborativo \\
\hline $\begin{array}{l}\text { Problemas } \\
\text { Proyectos } \\
\text { Experiencia } \\
\text { Contexto }\end{array}$ & $\begin{array}{l}\text { Interdisciplinario } \\
\text { Ejemplarizado } \\
\text { Teoría y práctica } \\
\text { Metodología de la investigación }\end{array}$ & $\begin{array}{l}\text { Equipos } \\
\text { Participación dirigida }\end{array}$ \\
\hline $\begin{array}{l}\text { El aprendizaje se organiza en } \\
\text { torno a problemas y se llevará a } \\
\text { cabo en los proyectos. } \\
\text { El problema es el punto de } \\
\text { partida para los procesos de } \\
\text { aprendizaje, coloca el aprendizaje } \\
\text { en contexto, y las bases del } \\
\text { aprendizaje en la experiencia del } \\
\text { alumno. } \\
\text { Basado en proyecto significa que } \\
\text { es una tarea que involucra análisis } \\
\text { de problema más complejo y } \\
\text { estrategias de resolución de } \\
\text { problemas. }\end{array}$ & $\begin{array}{l}\text { Se preocupa especialmente del } \\
\text { aprendizaje interdisciplinario, cruza los } \\
\text { límites entre temas relacionados y } \\
\text { métodos. } \\
\text { Es una práctica ejemplarizada en el } \\
\text { sentido de que el resultado del } \\
\text { aprendizaje es ejemplarizado para los } \\
\text { objetivos del plan de estudios. } \\
\text { Se apoya la relación entre la teoría y la } \\
\text { práctica. El proceso de aprendizaje usa } \\
\text { la teoría en el análisis de los problemas } \\
\text { y de los métodos de resolución de } \\
\text { problemas. }\end{array}$ & $\begin{array}{l}\text { En al aprendizaje en equipo, el } \\
\text { aprendizaje se lleva a cabo a través del } \\
\text { diálogo y la comunicación. Los } \\
\text { estudiantes no sólo aprenden el uno del } \\
\text { otro, sino que también aprenden a } \\
\text { compartir conocimientos y organizar por } \\
\text { sí mismos el proceso de aprendizaje. } \\
\text { También abarca el concepto de } \\
\text { aprendizaje dirigido por el participante, } \\
\text { lo que indica una propiedad colectiva } \\
\text { del proceso de aprendizaje y, sobre } \\
\text { todo, en la formulación del problema. }\end{array}$ \\
\hline
\end{tabular}

Tabla 2. Elementos del modelo II del ABP (Adaptado de Kolmos et. al (2009))

\begin{tabular}{|l|l|}
\hline Modelo II & Descripción \\
\hline & $\begin{array}{l}\text { Conocimiento: know-how } \\
\text { Aprendizaje: habilidades para el lugar de trabajo. } \\
\text { Aprendizaje basado en } \\
\text { problemas para la } \\
\text { acción profesional }\end{array}$ \\
& $\begin{array}{l}\text { Escenario del problema: la vida real. } \\
\text { Estudiantes: aprender a resolver problemas reales con el fin de emprender acciones prácticas } \\
\text { Evalitadores: demostración de habilidades prácticas }\end{array}$ \\
\hline
\end{tabular}

Tabla 3. Características de las actividades auténticas (Adaptado de Reeves et al. (2002))

\begin{tabular}{|l|l|}
\hline Características & Descripción \\
\hline Relevancia del mundo real & $\begin{array}{l}\text { Las actividades deben corresponderse a las actividades del mundo real de los } \\
\text { profesionales en vez de las actividades fuera del contexto o basadas en la sala de clase. }\end{array}$ \\
\hline Poco definida & $\begin{array}{l}\text { Las actividades requieren que los estudiantes definan las tareas y sub tareas necesarias } \\
\text { para cumplir la actividad. }\end{array}$ \\
\hline $\begin{array}{l}\text { Tareas complejas y } \\
\text { sostenidas en el tiempo }\end{array}$ & $\begin{array}{l}\text { Las actividades se cumplen en un plazo de días, semanas o meses en lugar de minutos } \\
\text { u horas. Requieren un aporte considerable de tiempo y recursos intelectuales. }\end{array}$ \\
\hline Perspectivas múltiples & $\begin{array}{l}\text { A los estudiantes se les brinda la oportunidad de examinar la tarea desde varias } \\
\text { perspectivas al usar una variedad de recursos y de identificar la información pertinente } \\
\text { de la que no lo es. }\end{array}$ \\
\hline Colaboración & $\begin{array}{l}\text { Para el desarrollo de la tarea, la colaboración figura como elemento integral y requerido } \\
\text { para cumplir con ésta. }\end{array}$ \\
\hline $\begin{array}{l}\text { En un contexto basado en } \\
\text { valores }\end{array}$ & \begin{tabular}{l} 
Ofrece a los estudiantes la oportunidad de reflexionar y aplicar sus creencias y valores. \\
\hline Interdisciplinario
\end{tabular} \\
\hline $\begin{array}{l}\text { Evaluado de una forma } \\
\text { auténtica }\end{array}$ & $\begin{array}{l}\text { Lastividades convocan perspectivas interdisciplinarias y permiten que los estudiantes } \\
\text { materia o dominio específico. }\end{array}$ \\
\hline Productos reales & $\begin{array}{l}\text { La evaluación se integra al aprendizaje de una manera que refleja la evaluación de } \\
\text { calidad del mundo profesional. }\end{array}$ \\
\hline $\begin{array}{l}\text { Múltiples resultados } \\
\text { posibles }\end{array}$ & $\begin{array}{l}\text { Las actividades auténticas generan productos terminados con un valor propio en vez de } \\
\text { un valor preparativo. }\end{array}$ \\
\hline
\end{tabular}

Así, se desarrolló la experiencia en el contexto del citado curso desde el año 2011 al presente. Durante su desarrollo (2015), se consideró pertinente hacer un primer acercamiento de tipo reflexivo, analizando y describiendo cómo la enseñanza en base al ABP (contextualizados en problemas reales de empresas) influencian el ejercicio de la profesión del Ingeniero Informático de la Universidad de Tarapacá. 
Tomando en consideración lo anterior, se revisó la literatura relacionada con la aplicación del ABP y se observó que la mayoría de las investigaciones apuntaban a mejorar ya sea la motivación de los alumnos, el rendimiento académico o hasta aumentar la persistencia en el estudio, pero no se encontraron estudios que vincularan el uso de ABP (proyectos reales en empresas reales) y su impacto en el ejercicio temprano de la profesión.

Por ejemplo, diversos trabajos dan cuenta de una serie de aplicaciones del ABP en la enseñanza superior (Davies et al. 2011; Martínez et al. 2011; van Hattum-Janssen et al. 2012; Aliakbarian et al. 2014), pero no dirigidos en el sentido de este trabajo. Otras investigaciones muestran que el ABP fomenta habilidades tan importantes como el aprendizaje autónomo, la capacidad de expresión oral y escrita, la capacidad de autoevaluación, la planificación del tiempo, o la planificación por proyectos. Además, mejora la motivación del alumno, lo que se traduce en un mejor rendimiento académico y una mayor persistencia en el estudio (Bará, 2003; Galeana, 2006; Jenkins y Lackey, 2005; Menéndez, 2003; Pérez et al., 2008 y Tippelt y Lindeman, 2001). Otros estudios se orientan al desarrollo de competencias específicas en estudiantes de ingeniería y al desempeño del docente al aplicar ABP (Benítez y García, 2013; Fernández y Duarte, 2013).

Respecto de la aplicación del ABP en la enseñanza de la Ingeniería Informática, vale la pena mencionar los estudios de (Gallardo et al. 2011; Song et al. 2011; Kellett, 2012; Pallejà et al. 2013; Soska et al. 2014; Indiramma, 2014; García et al. 2014; Kokosy et al. 2014; Naves et al. 2015). Un trabajo importante para destacar es el de (Astorga et al. 2015) ya que determina el impacto del ABP en el desarrollo de las competencias asociadas a los roles más representativos en la industria de software que después desempeñarán en su ejercicio profesional estudiantes de Licenciatura en Informática. Los estudiantes participan como personal en una empresa escolar, donde desarrollan proyectos que requieren soluciones reales.

De todo lo anteriormente expuesto se desprendió una cuestión principal: ¿Cómo se relaciona el desarrollo ABP (contextualizados en problemas reales de empresas) y el ejercicio temprano de la profesión por parte de exalumnos de una carrera de Ingeniería Informática?

\section{MÉTODO DE TRABAJO}

El método se presenta en dos subsecciones: en una se describe el modelo conceptual de la experiencia que incluye las etapas de definición, desarrollo y evaluación; en otra sección se describe a los participantes de la experiencia.

\section{Diseño conceptual}

El periodo de análisis de la experiencia va desde 2011 a 2017. La experiencia fue de naturaleza transversal y el método de recolección de datos primarios fue a través de la aplicación de una encuesta a exalumnos de la Universidad de Tarapacá que habían realizado el citado curso y que habían desarrollado software para micro empresas de la Provincia de Arica. Así mismo, se entrevistó a los profesores del curso y a los empresarios para los que los exalumnos desarrollaron los softwares. Como estrategia de control, se encuestó a un grupo de exalumnos que no realizaron el citado curso. Finalmente, al término del sexto año de la experiencia (2017), se consultó nuevamente a una muestra de exalumnos ya encuestados y a un experto en desarrollo de proyectos de software (empresario). Las especificaciones de la muestra de participantes se mencionan más adelante.

La experiencia inició el año 2011 por la necesidad de evaluar el logro de competencias de egreso del Ingeniero Informático de la UTA. Para ello se ideó la estrategia de cambiar el tipo de aplicaciones que desarrollaban los estudiantes en la etapa terminal de la carrera, desde aplicaciones asociadas a problemas ficticios o dentro del propio aparato administrativo de la UTA por problemas reales en empresas reales. Con estos lineamientos, se diseñó el modelo de trabajo para la experiencia que se muestra en la Fig. 1a.

Inicialmente, se establecieron las competencias de egreso a reforzar con esta estrategia y se instauró el uso del Aprendizaje Basado en Proyectos como enfoque pedagógico, definiendo una serie de características del mismo que ayudarían en esta dirección. Con el contexto metodológico definido, se estableció que el tipo de empresa a ser considerada serían Micro Empresas, por el gran número de ellas existentes en la Región de Arica y Parinacota (10.840) (Uriarte, 2013) las menos tecnológicas y dado que existen empresas de desarrollo de software de exalumnos que fundamentalmente sus clientes son las pequeñas empresas de la Región. Se diseñó una convocatoria y que fue en algunos casos por concurso público y en otros por invitación directa. 
Una vez realizada la convocatoria con un número definido de cupos, según el número de alumnos inscritos en el curso, se procedió al desarrollo de la experiencia, que sería replicada anualmente, desde el año 2011. Cabe destacar que las experiencias del 2016 y 2017, ya fueron realizadas, pero los datos serán recogidos el año 2018 o 2019. La experiencia inició con una planificación preliminar, describiendo a los estudiantes la forma de trabajo semestral y cómo será la evaluación. Luego, en una reunión conjunta con los empresarios y alumnos, se realizó la asignación de empresas de manera aleatoria. Así mismo, se establecieron los requisitos preliminares de los problemas a ser abordados, base de un convenio que fue firmado ante el Jefe de Carrera. Este convenio dejó explícito, condiciones, fechas, propiedad intelectual, y confidencialidad, entre otros.

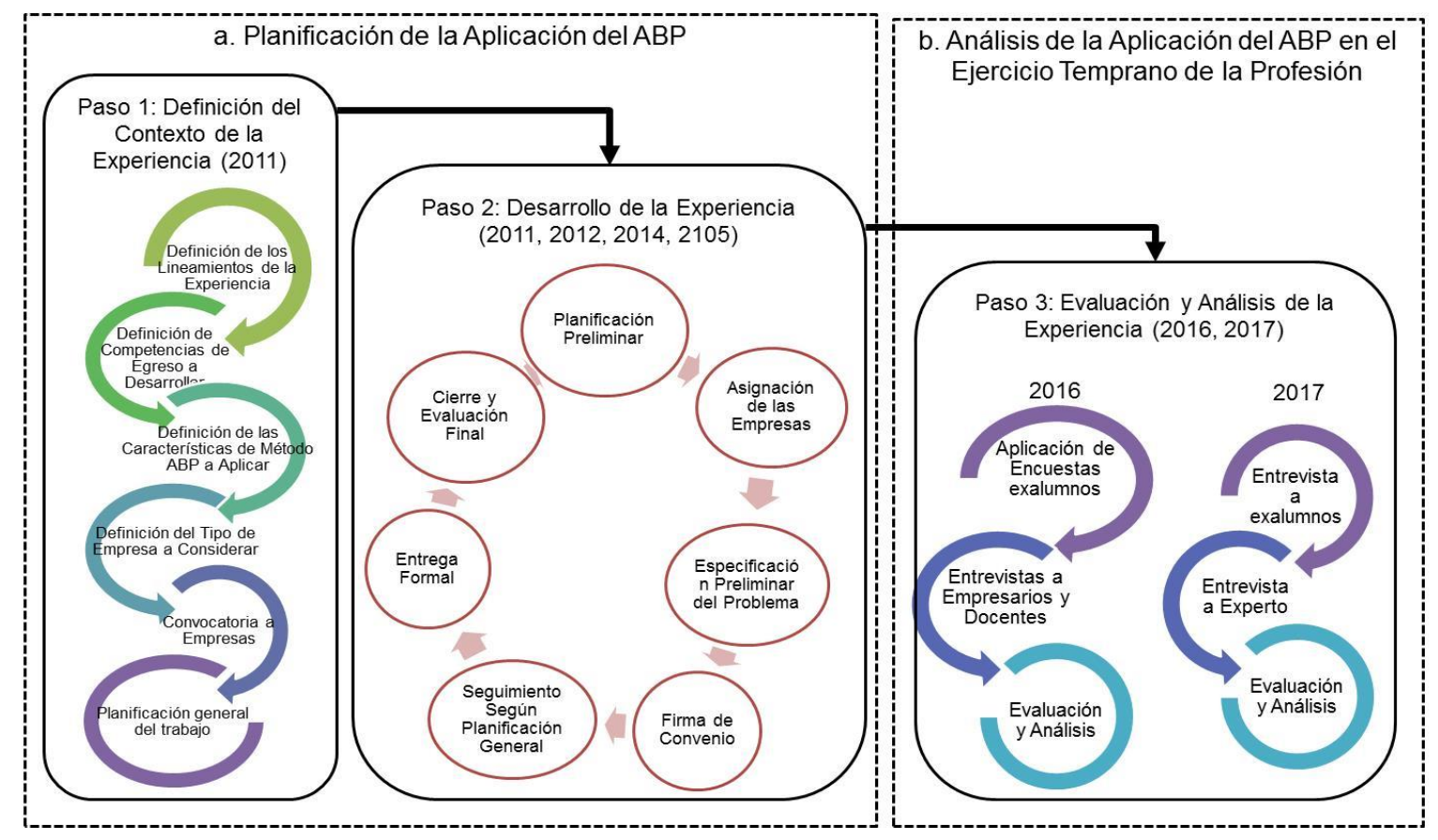

Fig. 1. Modelo Conceptual de la Experiencia

Firmados los respectivos convenios, se inicia un seguimiento según el plan de trabajo estándar, se realizan evaluaciones del proceso y resultados parciales de acuerdo a las fechas establecidas en el plan, actuando los profesores como facilitadores y el curso en su conjunto como un equipo que teniendo un plan de trabajo estándar desarrolla soluciones específicas para cada empresa. Cabe destacar que metodológicamente se sigue un mismo modelo de desarrollo de software, los mismo estándares tanto de producto como proceso. Concluidos los productos software que implementan los requisitos indicados en los convenios, se realiza una entrega formal por parte de los estudiantes, una capacitación y un manual de usuario, que el empresario debe evaluar indicando si cumplen o no con sus expectativas. Con esta evaluación y una presentación final, los profesores evalúan tanto el producto como el proceso realizado.

Como se había mencionado anteriormente, en el año 2015, durante el desarrollo de la experiencia (Fig. 1a), se consideró pertinente hacer un primer acercamiento de tipo reflexivo, analizando y describiendo cómo la enseñanza en base al ABP (contextualizados en problemas reales de empresas) influencian el ejercicio de la profesión. Así, una vez que los estudiantes egresaron y encontraban haciendo ejercicio de la profesión temprana, se les encuestó para realizar una evaluación de esta influencia (2016). Así mismo, se realiza una entrevista a cada empresario (relacionado a cada exalumno) para evaluar el nivel de satisfacción con el trabajo del exalumno asignado a su empresa. Con los datos recogidos, se procede a su tabulación y análisis que se describe más adelante. Cabe destacar que en este reporte, no se consideran las experiencias 2016 y 2017 ya que los alumnos aún no llevan 1 año fuera de la carrera o haciendo ejercicio de la profesión. El método para esta evaluación se describe en la Fig. 1b.

\section{Muestra de Participantes}

El curso "Taller de Proyectos de Software" de la Carrera de Ingeniería Civil en Computación e Informática (ICCI) de la UTA se imparte durante el décimo semestre ( $5^{\circ}$ año). Tiene 8 horas pedagógicas semanales, cada semestre tiene 16 semanas. También tiene como curso previo "Ingeniería de Software". Finalmente, tiene como objetivo general: "Desarrollar y documentar un proyecto de software utilizando herramientas de Ingeniería de Software, Modelo de Proceso, Herramientas que den soporte al Proceso de Desarrollo, Herramientas y Métodos para desarrollar una Interfaz Gráfica de Usuario". Cada alumno desarrolla un software para cada empresa. En promedio han participado 7 a 8 estudiantes por año. 
Por otra parte, solo se consideraron los resultados de la aplicación de las experiencias de los años 2011, 2012, 2014 y 2015 ya que el año 2013 no se dictó el curso con el enfoque en análisis y los del año 2016 en adelante, aún no cumplen el requisito que los estudiantes tengan un año de egreso o ejercicio temprano de la profesión. Así, el total de exalumnos que participó del análisis, fue de 21. Así mismo, para el grupo de exalumnos que no participaron del curso con uso de ABP, se consideraron aquellos anteriores al año 2011 y los del año 2013, siendo en total 8. Los profesores son los que han dictado el curso desde 2011 con el nuevo enfoque (3). Los empresarios quedan determinados por los exalumnos, ya que son para quiénes les fuera desarrollado el software. Finalmente, los exalumnos entrevistados (4) son los que respondieron a la invitación y que están ejerciendo en Santiago de Chile, donde se tiene un volumen y tamaño de los proyectos de desarrollo de software importante. El experto se seleccionó considerando su amplia experiencia en el desarrollo de proyectos de desarrollo de software (más de 20 años) y ha sido empleador de más de 50 exalumnos de la carrera ICCI.

La encuesta contó de 3 secciones. La primera sección, requiere del exalumno, evaluar la importancia de cada característica del modelo ABP (tabla 3) y el nivel que él daría a cada una de ellas en la repetición del curso. En la Fig. 2a, se puede apreciar el encabezado y las 3 primeras características del ABP evaluadas. La segunda sección, evaluar el impacto del curso aplicando ABP definido, en su ejercicio profesional temprano (Fig. 2b). Finalmente, se le solicitó explicar brevemente cuál creyó fue el mayor aporte del uso del ABP definido en su ejercicio temprano de la profesión (Fig. 2c).

a.

\begin{tabular}{|c|c|c|c|c|c|c|c|}
\hline \multirow{2}{*}{$\begin{array}{l}\text { CARACTERISTICA } \\
\text { DE LOS } \\
\text { PROYECTOS } \\
\text { REALIZADOS PARA } \\
\text { UNA PYME }\end{array}$} & \multirow[t]{2}{*}{ DESCRIPCIÓN } & \multicolumn{3}{|c|}{$\begin{array}{l}\text { AHORA COMO PROFESIONAL: } \\
\text { ¿CÓMO EVALUAA LA IMPORTANCIA DE CADA } \\
\text { CARACTERISTIICA DE LOS PROYECTOS } \\
\text { REALIZADOS PARA PYME? }\end{array}$} & \multicolumn{3}{|c|}{$\begin{array}{l}\text { AHORA COMO PROFESIONAL: } \\
\text { ¿QUÉ NIVEL DE ÉNFASIS DARIAA A CADA } \\
\text { CARACTERISSTICA DE LOS PROYECTOS } \\
\text { REALIZADOS PARA PYME? }\end{array}$} \\
\hline & & $\begin{array}{c}\text { NO } \\
\text { RELEVANTE }\end{array}$ & $\begin{array}{l}\text { MEDIANAMENTE } \\
\text { RELEVANTE }\end{array}$ & $\begin{array}{c}\text { MUY } \\
\text { RELEVANTE }\end{array}$ & DISMINUIR & MANTENER & AUMENTAR \\
\hline $\begin{array}{l}\text { Relevancia al mundo } \\
\text { real }\end{array}$ & $\begin{array}{l}\text { Las actividades deben } \\
\text { corresponderse a las actividades } \\
\text { del mundo real de los } \\
\text { profesionales en vez de las } \\
\text { actividades fuera del contexto o } \\
\text { basadas en la sala de clase. }\end{array}$ & & & $\mathrm{x}$ & & & $\mathrm{x}$ \\
\hline Poco definido & $\begin{array}{l}\text { Las actividades requieren que los } \\
\text { estudiantes definan las tareas y } \\
\text { sub tareas necesarias para cumplir } \\
\text { la actividad. }\end{array}$ & & & $\mathrm{x}$ & & & $\mathrm{x}$ \\
\hline
\end{tabular}

b.

INDIQUE EL IMPACTO EN SU EJERCICIO PROFESIONAL, EL HABER DESARROLLADO EN LA ASIGNATURA DE "TALLER DE PROYECTOS DE SOFTWARE" UN PROYECTO REAL CON UNA EMPRESA REAL (PYME) NO RELEVANTE MUY RELEVANTE

C.

C) EXPLIQUE BREVEMENTE CUAL CREE USTED FUE EL MAYOR APORTE DEL DESARROLLO DE ESTE TIPO DE PROYECTO EN EL EJERCICIO DE SU PROFESIÓN (POR EJEMPLO INDIQUE QUE MÁS HA APLICADO)

Fig. 2. Estructura de la Encuesta

\section{Técnica de análisis de datos}

La técnica de análisis de datos fue mediante el procesamiento de la encuesta, obteniendo como estadísticos: promedios y frecuencias. También, se procesaron las entrevistas realizadas a exalumnos, profesores y experto.

\section{RESULTADOS Y DISCUSIÓN}

Los resultados de la experiencia han sido muy positivos en todos los aspectos. En primer lugar, solo uno de los proyectos no fue concluido, es decir, el $99,9 \%$ de los proyectos fueron concluidos satisfactoriamente y las evaluaciones por parte de los empresarios fueron muy positivas. En la tabla 4 se muestran todos los proyectos abordados en los distintos años (29). Como se comentó, para los años 2016 y 2017 aún no se obtienen los datos.

\section{Opinión de los exalumnos}

Los exalumnos que participaron del curso ABP definido (que se denominarán ExABP) que contestaron la encuesta fueron 21 de 29 , es decir un $72,4 \%$. Los exalumnos que no participaron del curso ABP definido (que se denominarán ExNABP) y que fueron contactados son 8, de los cuales el $100 \%$ contestó la encuesta. La encuesta considera la importancia que los ExABP dan a las características asociadas con el tipo de proyectos y el nivel de impacto en su ejercicio profesional temprano. A los ExNABP, que no 
realizaron proyectos de esta naturaleza, se les pregunta cuáles serían sus respuestas si hubieran realizado los proyectos asociados con PYMES. La encuesta consideró los tres aspectos señalados en la Fig. 2. En las siguientes secciones, se describen los resultados asociados a cada aspecto.

Tabla 4. Proyectos abordados desde 2011-2015

\begin{tabular}{|c|c|c|c|}
\hline 2011 - Marzo-Agosto & 2012 - Marzo-Agosto & 2014 - Agosto-Diciembre & 2015 - Marzo-Agosto \\
\hline $\begin{array}{l}\text { Gespack: Software para } \\
\text { apoyar la gestión de } \\
\text { envíos, clientes y servicios }\end{array}$ & $\begin{array}{l}\text { SONAR: Sistema de } \\
\text { gestión financiera para } \\
\text { emisoras radiales }\end{array}$ & $\begin{array}{l}\text { SISCON: Gestión de } \\
\text { Alumnos y Encuestas para } \\
\text { una empresa en educación }\end{array}$ & $\begin{array}{l}\text { Sistema Web Gestión de } \\
\text { Gestión Confecciones: } \\
\text { Cienyconfecciones }\end{array}$ \\
\hline $\begin{array}{l}\text { SIGECLI: Sistema Web } \\
\text { para la Gestión de } \\
\text { Clientes de la empresa } \\
\text { Corretaje Propiedades }\end{array}$ & $\begin{array}{l}\text { Sistema de Planificación } \\
\text { de Documentación } \\
\text { Financiera }\end{array}$ & $\begin{array}{l}\text { SWGCKC: Sitio Web Gestor } \\
\text { de Contenidos de K } \\
\text { Consultores }\end{array}$ & $\begin{array}{l}\text { Sistema Web Gestión de } \\
\text { Gestión de Eventos: hijos de } \\
\text { la música }\end{array}$ \\
\hline $\begin{array}{l}\text { SACFT: Sistema de Apoyo } \\
\text { para Centro de Formación } \\
\text { Técnica Tarapacá }\end{array}$ & $\begin{array}{l}\text { Sistema de gestión de } \\
\text { servicios Salón de Belleza }\end{array}$ & $\begin{array}{l}\text { Sistema de Gestión de } \\
\text { servicios de seguridad online }\end{array}$ & $\begin{array}{l}\text { Sistema Web Gestión de } \\
\text { Confecciones Susana }\end{array}$ \\
\hline $\begin{array}{l}\text { Sistema de Gestión de } \\
\text { Productos E.C.R. }\end{array}$ & Sistema IBIZZ & $\begin{array}{l}\text { Sistema de Inventariado JJ } \\
\text { \& SS Ingeniería y } \\
\text { Construcción }\end{array}$ & $\begin{array}{l}\text { Sistema Web Gestión Clean } \\
\text { Planet Ltda. Empresa } \\
\text { dedicada al aseo industrial }\end{array}$ \\
\hline $\begin{array}{l}\text { Sistema de inventario web } \\
\text { para una Fundación }\end{array}$ & $\begin{array}{l}\text { Sistema de gestión de } \\
\text { inventario y órdenes de } \\
\text { trabajo de la empresa }\end{array}$ & $\begin{array}{l}\text { Sistema de Control de } \\
\text { Insumos Juguería }\end{array}$ & $\begin{array}{l}\text { Sistema Web Gestión de } \\
\text { Confecciones Tina Fashion }\end{array}$ \\
\hline $\begin{array}{l}\text { ASOF: Gestión de las } \\
\text { órdenes de trabajo y } \\
\text { facturas de una Imprenta }\end{array}$ & $\begin{array}{l}\text { SAFCA: Sistema De } \\
\text { Administración Financiera }\end{array}$ & $\begin{array}{l}\text { RAYNA: Aplicación web para } \\
\text { empresa Rayito-Restaurante }\end{array}$ & $\begin{array}{l}\text { Sistema para la gestión de } \\
\text { costos y beneficios de } \\
\text { proyectos- Empresa: } \\
\text { Ingenieros consultores } \\
\text { proyecto norte limitada }\end{array}$ \\
\hline \multirow[t]{3}{*}{$\begin{array}{l}\text { SISFIB: Gestión de Datos } \\
\text { Ferretería }\end{array}$} & $\begin{array}{l}\text { Sistema de Gestión Admi- } \\
\text { nistrativa Clínica de Salud }\end{array}$ & $\begin{array}{l}\text { Sistema Web para } \\
\text { Pastelería y Amasandería }\end{array}$ & \\
\hline & & $\begin{array}{l}\text { Sistema de Gestión puesto } \\
\text { feria itinerante }\end{array}$ & \\
\hline & & $\begin{array}{l}\text { Crava Online-Salón de } \\
\text { Cosmética }\end{array}$ & \\
\hline
\end{tabular}

\section{a) Respecto de las características de los proyectos y el impacto en su ejercicio profesional}

Como se muestra en la Fig. 3, los ExABP, consideran en general todas las características asociadas con este tipo de proyecto como medianamente o muy relevante, destacándose la "relevancia al mundo real", "tareas complejas y sostenidas en el tiempo", "colaboración", "interdisciplinario" y "productos reales" $(76 \%, 81 \%, 86 \%$, $76 \%$ y $67 \%$ respectivamente). Por el contrario, sólo un bajo porcentaje considera no relevante las características "poco definido" y "perspectivas múltiples", (5\% y $5 \%$ respectivamente). Considerando simultáneamente los niveles medianamente y muy relevante, en general los ExABP perciben como importantes cada una de las características del tipo de proyecto que desarrollaron, para el ejercicio de su profesión.

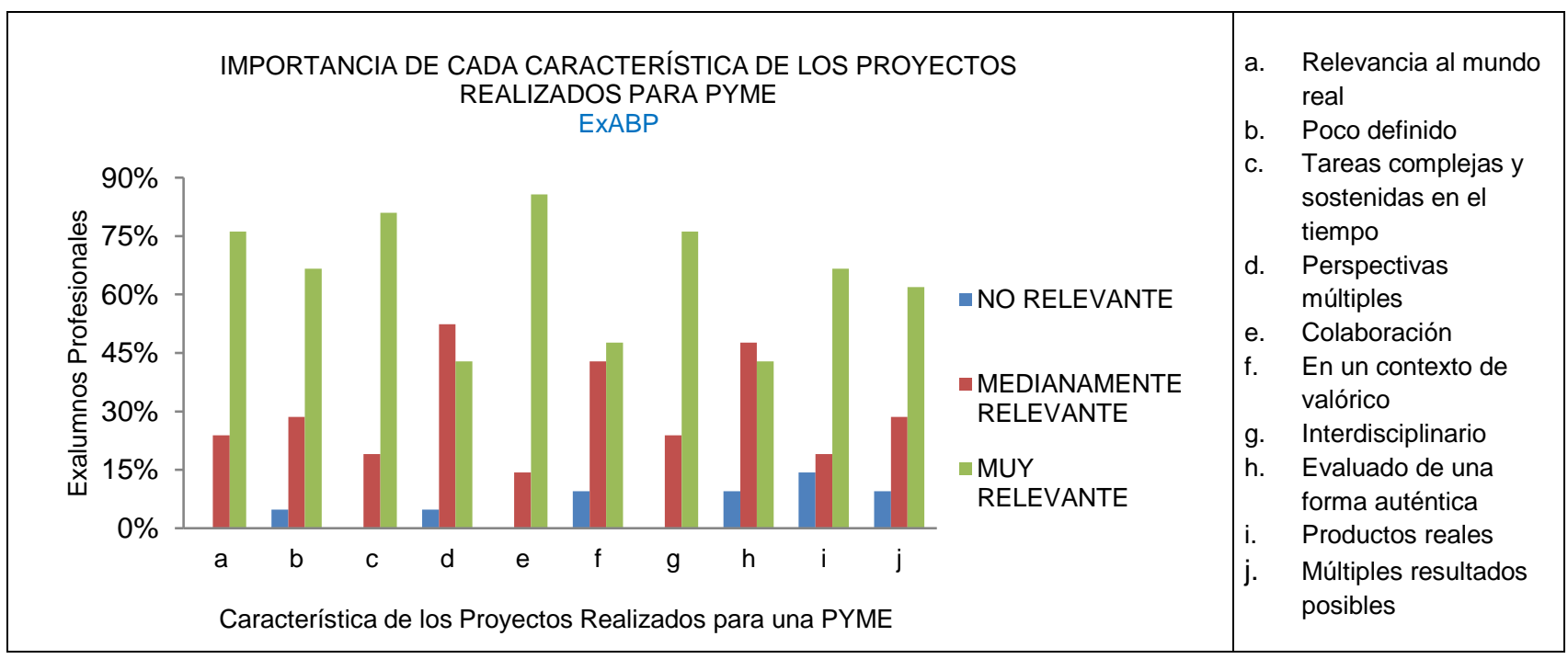

Fig. 3. Respuestas de los ExABP respecto de las características de los proyectos y el impacto en su ejercicio profesional 
Por su parte, a los ExNABP, se les preguntó, si los proyectos fueran reales y para PYMES qué importancia les darían a las características y sus respuestas fueron como se muestra en la Fig. 4. Los ExNABP, consideraron en general todas las características asociadas con este tipo de proyecto como medianamente o muy relevante, destacándose la "relevancia al mundo real", "tareas complejas y sostenidas en el tiempo", "colaboración" y "múltiples resultados posible" ( $88 \%, 75 \%, 88 \%$ y $75 \%$ respectivamente). Por el contrario, sólo un bajo porcentaje consideró no relevante las características "poco definido" y "productos reales" (13\% y $13 \%$ respectivamente). Considerando simultáneamente los niveles medianamente y muy relevante, en general los ExNABP percibieron como importantes cada una de las características del tipo de proyecto que desarrollarían, para el ejercicio de su profesión.

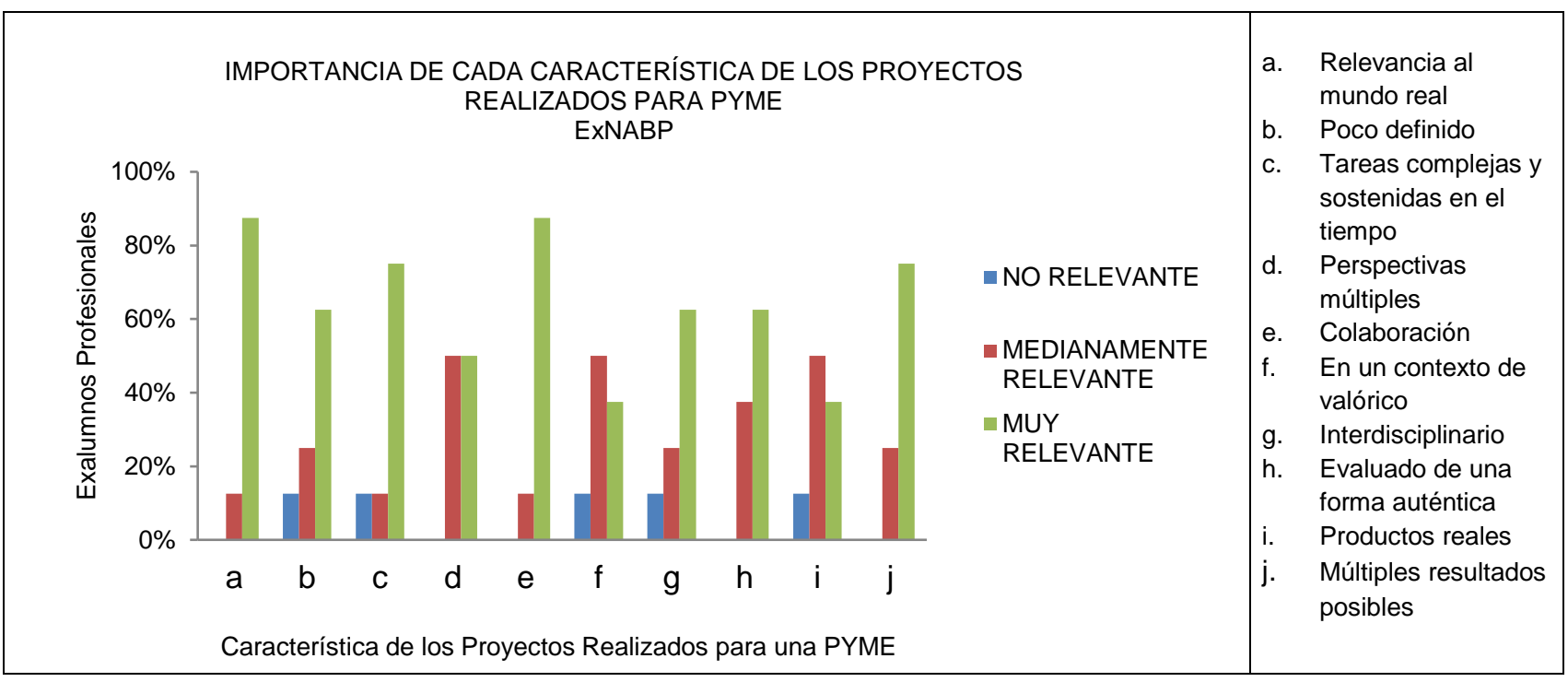

Fig. 4. Respuestas de los ExNABP respecto de las características de los proyectos y el impacto en su ejercicio profesional

Es importante destacar que no existen diferencias significativas entre los grupos ExNABP y ExABP, si se consideran simultáneamente los niveles medianamente y muy relevante. Pero si hay una diferencia entre los grupos si se observa la característica "productos reales", ya que en el caso de los ExABP, el $67 \%$ lo consideró muy relevante frente a un $38 \%$ de los ExNABP. Esto se explica porque los ExNABP no realizaron proyectos reales con las PYMES y no tienen la misma percepción que los ExABP. También, es interesante que ambos grupos no vieron con el mismo nivel de importancia el tema basado en valores ya que en los ExABP, el $48 \%$ le asigna un nivel muy relevante frente al 38\% de los ExNABP. Esto sería preocupante si ambos grupos lo consideraran no relevante.

También, a los ExABP se les preguntó sobre el nivel de énfasis que le darían a cada característica de los proyectos realizados para PYME, teniendo en cuenta el ejercicio de la profesión. Coherentemente (Fig. 5), los ExABP, señalaron que debe aumentarse el énfasis en las características de "relevancia al mundo real", "colaboración" e "interdisciplinario" (67\%, 67\% y 62\% respectivamente). Por otra parte, un bajo porcentaje de ellos consideró que debe disminuirse el énfasis en las características tales como "tareas complejas y sostenidas en el tiempo", "productos reales" y "múltiples resultados posibles" $(10 \%, 10 \%$ y $10 \%$ respectivamente). Considerando simultáneamente los niveles medianamente y muy relevante, en general los ExABP consideraron que no debe disminuirse cada una de las características del tipo de proyecto que desarrollaron, para el ejercicio de su profesión.

Por su parte, a los ExNABP se les preguntó si los proyectos que se hubieran realizado en el citado curso fueran reales y para PYMES qué énfasis les darían a las características y sus respuestas fueron como se muestran en la Fig. 6. También, los ExNABP, coherentemente (Fig. 6), señalaron que debe aumentarse el énfasis en las características de "relevancia al mundo real", "tareas Complejas y sostenidas en el tiempo", "colaboración" e "interdisciplinario" (75\%, 63\%, 63\% y 63\% respectivamente). Por otra parte, un bajo porcentaje de ellos consideró que debe disminuirse el énfasis en las características tales como "tareas complejas y sostenidas en el tiempo" y "en un contexto basado en valores" (25\% y $13 \%$ respectivamente). Tomando en cuenta simultáneamente los niveles medianamente y muy relevante, en general los ExNABP consideraron que no debe disminuirse cada una de las características del tipo de proyecto que desarrollaron, para el ejercicio de su profesión. De los datos se puede indicar que tampoco existen diferencias significativas entre los grupos en esta cuestión, si se consideran simultáneamente los niveles medianamente y muy relevante. Pero sí hay una diferencia entre los grupos si se observa la característica "evaluado de una forma auténtica". El grupo ExABP consideró que se debe mantener (81\%) y aumentar solo 
el (19\%) frente al grupo ExNABP que consideró se debería mantener $(50 \%)$ y aumentar el $(50 \%)$. Nuevamente, esto se explica porque los ExNABP no fueron evaluados de forma auténtica (la evaluación final de los ExABP fue considerando la satisfacción del empresario) que queda de manifiesto cuando señalan que la característica "relevancia al mundo real" debe ser aumentada por un $75 \%$ de ellos. Reiteradamente, es interesante que ambos grupos no vieran con el mismo nivel de importancia a la característica basado en valores ya que de los ExABP, el $29 \%$ dice que hay que aumentarlo frente al $38 \%$ del grupo ExNABP.

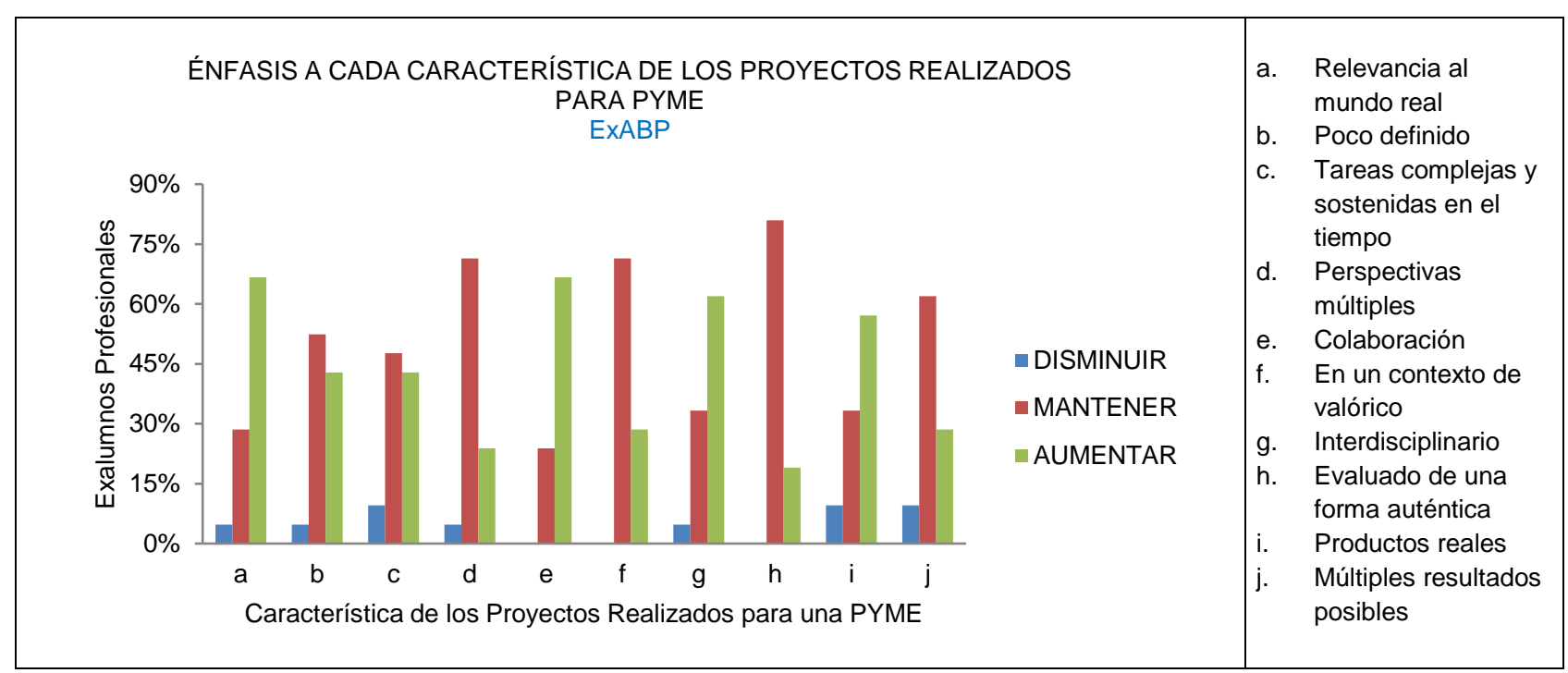

Fig. 5. Respuestas de los ExABP respecto del énfasis que le daría a las características de los proyectos y el impacto en su ejercicio profesional

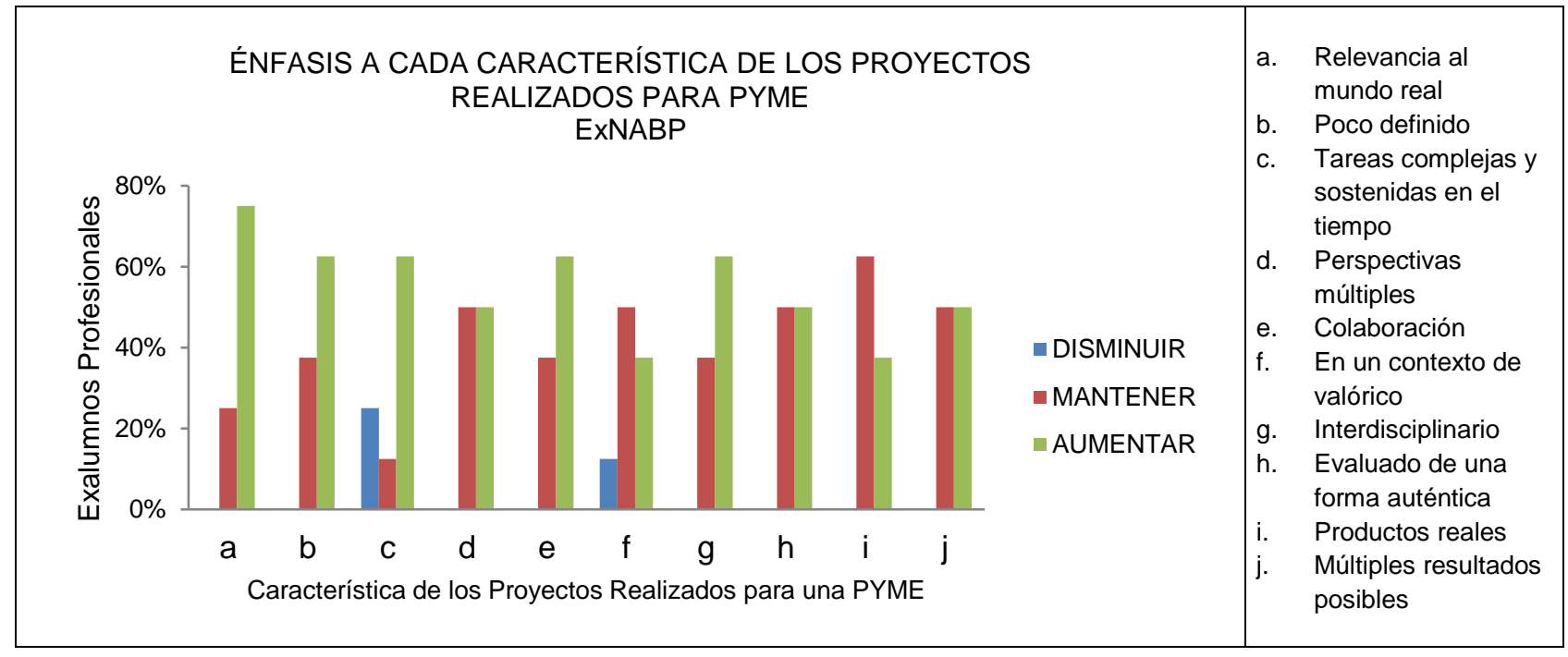

Fig. 6. Respuestas de los ExNABP respecto del énfasis que le daría a las características de los proyectos y el impacto en su ejercicio profesional

\section{b) Respecto del uso de proyectos reales y el impacto en su ejercicio profesional}

Como se muestra en la Fig. 7, el $90 \%$ de los ExABP, consideró que el uso de este tipo de proyecto en su formación ha tenido un impacto ya sea muy relevante (71\%) o un impacto medianamente relevante (19\%) en el ejercicio de su profesión.

Con respecto de los ExNABP, como se muestra en la Fig. 8, el 100\% consideró que el uso de este tipo de proyecto, en su formación, habría tenido un impacto ya sea muy relevante (62\%) o un impacto medianamente relevante (38\%) en el ejercicio de su profesión. Sin embargo, en el grupo ExNABP nadie consideró "no relevante" el impacto en el ejercicio de su profesión el desarrollar este tipo de proyectos, frente al $10 \%$ del grupo ExABP. Realizando un análisis en detalle de esta situación, se descubrió que los 2 ExABP (10\%) no tenían los prerrequisitos necesarios para realizar adecuadamente el curso, lo que habría influido en su respuesta, ya que señalaron que los esfuerzos realizados para alcanzar el éxito fueron muy grandes, tanto así que no le permitieron lograr las experiencias que sus otros compañeros obtuvieron. El 
hecho de cursar la asignatura sin sus pre-requisitos, no fue conocido hasta la realización del análisis, pero además no depende de los profesores del curso, sino de un jefe de estudios.

IMPACTO EN SU EJERCICIO PROFESIONAL EXABP

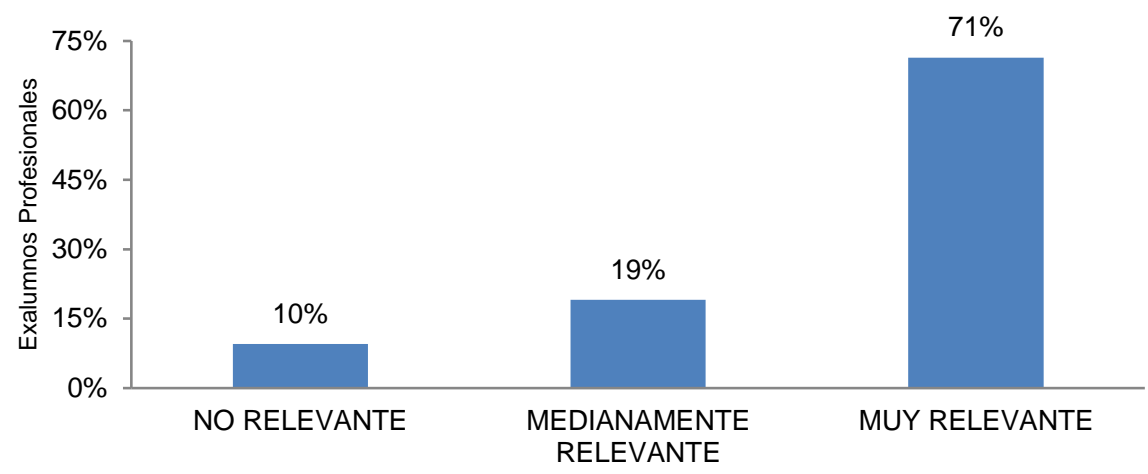

Nivel de Impacto

Fig. 7. Respuestas de los ExABP respecto del uso de proyectos reales y el impacto en su ejercicio profesional

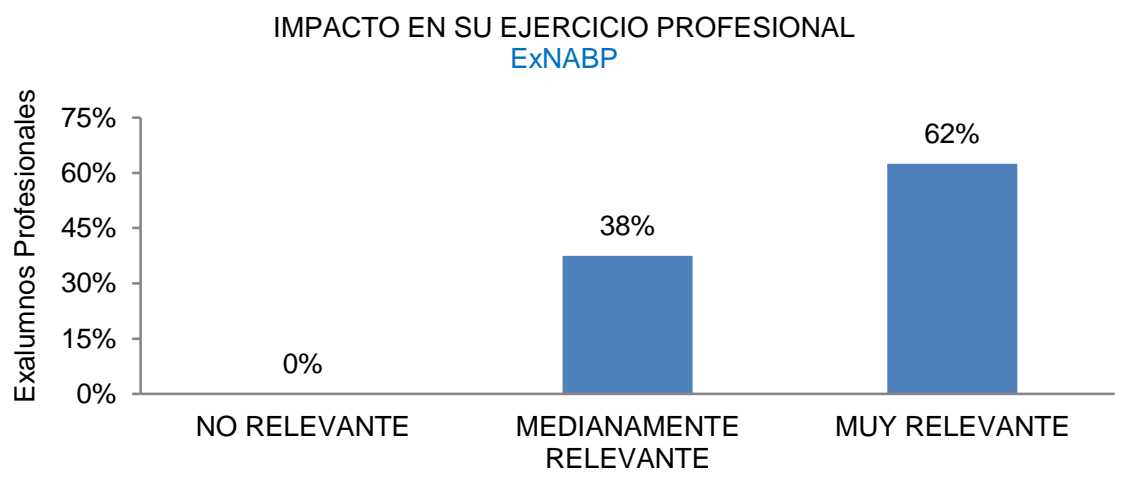

Nivel de Impacto

Fig. 8. Respuestas de los ExNABP respecto del uso de proyectos reales y el impacto en su ejercicio profesional

c) Explique brevemente cuál cree usted fue el mayor aporte del desarrollo de este tipo de proyecto en el ejercicio de su profesión

Con respecto al mayor aporte de la experiencia en el ejercicio de la profesión, en la tabla 5 se puede apreciar un resumen de los comentarios de los ExABP. Así mismo, en la tabla 6 se presentan los comentarios de los ExNABP. Es importante destacar que en general los ExABP, consideraron que este tipo de proyecto les aporta experiencia previa en la captura de necesidades y requerimientos de empresas 0 clientes $(42,9 \%)$, nivelar aspectos no cubiertos previamente en la carrera $(33,3 \%)$, entre otros. Con respecto de los ExNABP, consideraron que este tipo de proyecto les aportaría experiencia previa en en la captura de necesidades y requerimientos de empresas o clientes (37,5\%), nivelar aspectos no cubiertos previamente en la carrera $(37,5 \%)$, romper la barrera entre la teoría y lo real $(37,5 \%)$, ajustarse a una planificación $(50,0 \%)$, entre otros. No se observan diferencias significativas, por el contrario, hay un aumento en el grupo ExNABP, respecto de la planificación y emerge como relevante el trabajar en equipos.

Tabla 5. Apreciaciones de los ExABP

\begin{tabular}{|l|c|c|}
\hline \multicolumn{1}{|c|}{ Aporte } & Frecuencia & $\%$ \\
\hline Comprender necesidades de una empresa o cliente & 9 & $42,9 \%$ \\
\hline $\begin{array}{l}\text { Nivelar aspectos técnicos y personales no cubiertos en la } \\
\text { formación universitaria }\end{array}$ & 7 & $33,3 \%$ \\
\hline Romper la barrera entre la teoría y lo real & 2 & $9.5 \%$ \\
\hline Enfrentar problemas reales diversos con soluciones diferentes & 2 & $9.5 \%$ \\
\hline Conocer mejor las capacidades personales & 2 & $9.5 \%$ \\
\hline Ajustarse a una planificación & 2 & $9.5 \%$ \\
\hline
\end{tabular}


Tabla 6. Apreciaciones de los ExNABP

\begin{tabular}{|l|c|c|}
\hline \multicolumn{1}{|c|}{ Aporte } & Frecuencia & $\%$ \\
\hline Comprender necesidades de una empresa o cliente & 3 & $37,5 \%$ \\
\hline $\begin{array}{l}\text { Nivelar aspectos técnicos y personales no cubiertos en la } \\
\text { formación universitaria }\end{array}$ & 3 & $37,5 \%$ \\
\hline Romper la barrera entre la teoría y lo real & 3 & $37,5 \%$ \\
\hline Enfrentar problemas reales diversos con soluciones diferentes & 1 & $12,5 \%$ \\
\hline Ajustarse a una planificación & 4 & $50,0 \%$ \\
\hline Trabajar en equipo & 1 & $12,5 \%$ \\
\hline
\end{tabular}

\section{Opinión de los empresarios}

Como fue mencionado, la opinión en general de los empresarios beneficiarios, fue positiva. Opinaron que el trabajo es de calidad y con productos útiles para sus negocios. Solo en uno de los 29 proyectos considerados en este estudio, no se concluyó con el software requerido. En la tabla 7 , se resumen los comentarios de los empresarios respecto de la experiencia.

Tabla 7. Apreciaciones de los empresarios

\begin{tabular}{|l|}
\hline \multicolumn{1}{|c|}{ Comentario } \\
\hline "... lo instalamos esta semana y es de gran ayuda porque ahora está todo por sistema y no hay \\
Archivadores y eliminamos el papeleo. Estamos muy agradecidos de la UTA". \\
\hline $\begin{array}{l}\text { "para ampliar el sistema de pedidos a través de Internet... ahora contamos con exhibición de productos, manejo de } \\
\text { inventario, más independencia de la oficina". }\end{array}$ \\
\hline $\begin{array}{l}\text { "Es una tremenda ayuda porque teníamos todo el registro con el método antiguo y cuando venían nuestros clientes, que } \\
\text { son rotativos, nos costaba encontrar sus antecedentes", señaló el propietario. "Recibí el ofrecimiento de la UTA e }\end{array}$ \\
\hline "El trabajo del alumno fue un aporte y la calidad del mismo fue genial". \\
\hline "Recibí un trabajo de calidad y que nos ayudó mucho en nuestras tareas cotidianas" \\
\hline "El alumno desarrolló un software completo y muy útil para nosotros, estamos muy agradecidos" \\
\hline $\begin{array}{l}\text { "Buen trabajo, consistente y asistencia permanente. Tenemos estadísticas periódicas, honorarios, etc. Importante es } \\
\text { que aún tenemos contacto con el alumno". }\end{array}$ \\
\hline "Lamentablemente, el trabajo no fue concluido, aunque el alumno era proactivo y capaz" \\
\hline $\begin{array}{l}\text { "La calidad del desarrollo de sistema por parte alumno, fue excelente, respondiendo a nuestros requerimientos } \\
\text { planteados. Se indica que cumplió con las fechas estipuladas para las entrevistas y toma de requerimientos, como de } \\
\text { demostración y entrega de sistema a la unidad de capacitación. El alumno es una persona muy capaz". }\end{array}$ \\
\hline $\begin{array}{l}\text { "La percepción que se tuvo en el momento de implementación de dicho programa fue alta. El producto final fue } \\
\text { excelente". }\end{array}$ \\
\hline
\end{tabular}

Tabla 8. Apreciaciones de los docentes

\begin{tabular}{|c|l|}
\hline Aspecto & \multicolumn{1}{c|}{ Comentario } \\
\hline Práctica & $\begin{array}{l}\text { "Aunque en todas las asignaturas o cursos de la especialidad, se considera la realización de prácticas } \\
\text { de programación, diseño, implementación, etc. en muchas ocasiones los estudiantes se quejaban de la } \\
\text { falta de conexión entre la teoría y la práctica. Esta estrategia ha ayudado a disminuir } \\
\text { considerablemente esta apreciación por parte de los estudiantes." }\end{array}$ \\
\hline Autonomía & $\begin{array}{l}\text { "En cada versión de la experiencia se ha ido aumentando considerablemente la autonomía de los } \\
\text { estudiantes. En la primera versión del año 2011, en casi todo el proceso se fue conduciendo al } \\
\text { estudiante, sin embargo, durante la versión del 2016, sólo se entregó un plan de trabajo genérico con } \\
\text { hitos definidos para las entregas parciales y evaluaciones parciales. Dejando el proceso completo de } \\
\text { cargo de los estudiantes. Aunque en la primera estrategia hay mayor trabajo por parte del docente, en } \\
\text { la última, hay una mayor incertidumbre, acercándose más al mundo real." }\end{array}$ \\
\hline
\end{tabular}


Tabla 8 (continuación)

\begin{tabular}{|l|l|}
\hline $\begin{array}{l}\text { Problemas } \\
\text { reales }\end{array}$ & $\begin{array}{l}\text { "Trabajar con usuarios reales con vicisitudes emergentes, dudas, puntos de vista, exigencias, cambio, } \\
\text { etc. enfrenta al estudiante con situaciones complejas donde deben movilizar o desplegar varias } \\
\text { competencias al mismo tiempo, sean estas profesionales o genéricas. Deben tomar de decisiones." }\end{array}$ \\
\hline Calidad & $\begin{array}{l}\text { "Muchos estudiantes llegan a los cursos finales, insistiendo que la calidad está en el producto que ellos } \\
\text { construyeron, pero al final, se dan cuenta, que quién define la calidad del producto es el cliente en } \\
\text { función del logro de sus requerimientos iniciales." }\end{array}$ \\
\hline Colaboración & $\begin{array}{l}\text { "Aunque cada estudiante enfrenta una situación distinta (empresa/problema), el proceso de producción } \\
\text { es el mismo y la planificación del trabajo es genérica, el estudiante debe colaborar con su cliente y con } \\
\text { sus pares para mejorar sus resultados continuamente." }\end{array}$ \\
\hline $\begin{array}{l}\text { Productos y } \\
\text { Reales }\end{array}$ & $\begin{array}{l}\text { "Uno de los aspectos que más he discutido son las ventajas y desventajas del trabajo en grupo y en } \\
\text { equipo por parte del estudiante. Uno de los aspectos difíciles de controlar es que el aprendizaje y } \\
\text { experiencias sean homogéneos, es decir que el trabajo en grupo enmascare que uno o más alumnos, } \\
\text { avancen sin tener los conocimientos o habilidades mínimas. Esta estrategia, enfrenta individualmente a } \\
\text { cada estudiante con un problema real, debe seguir un proceso de desarrollo y entregar a un cliente un } \\
\text { producto completo y satisfactorio. Sin embargo, debe presentar y defender resultados parciales ante } \\
\text { un grupo de pares y los docentes de manera continua, permitiéndole recibir retroalimentación sobre el } \\
\text { proceso y producto." }\end{array}$ \\
\hline
\end{tabular}

\section{Opinión de los docentes}

Por su parte, en la tabla 8 , se muestran los comentarios de los docentes a cargo de la experiencia durante estos años. Esta experiencia ha dado, por una parte, solución a peticiones de los alumnos por trabajos más reales y experimentales y por otra, a las exigencias del programa, para que los egresados sean capaces e idóneos para el desarrollo efectivo y eficaz de software.

\section{Opinión de los ExABP nuevamente consultados}

Cuatro ExABP ya encuestados fueron entrevistados (los que respondieron a la invitación y que están ejerciendo en Santiago de Chile). En general, confirmaron sus respuestas anteriores, pero aportaron las observaciones indicadas en la tabla 9. En resumen, es destacable que los entrevistados, consideraron que las características que convendría mantener en los proyectos es el trabajo en equipo. Otras fueron la complejidad del problema, el uso de herramientas de desarrollo utilizadas en la industria en la actualidad, y la investigación sobre tecnologías aplicables.

Tabla 9. Resumen de las observaciones realizadas por los entrevistados

\begin{tabular}{l} 
Resumen observaciones \\
\hline Los problemas deben seguir siendo del mundo real. \\
Desarrollar capacidad de la programación día a día y seguirla. \\
Comprensión que las horas son dinero del Cliente que paga el desarrollo, más cuando trabajas para una empresa. \\
Debe hacerse más patente el tema de la ética y los valores que se ponen en juego a la hora de desarrollar una solución \\
para un cliente. El cliente siempre solicitará todo para el día y hora que fue planificado, no lo importará cómo se hace. \\
Los proyectos reales ayudan a enfrentar problemas que jamás se creía que existían. En la realidad eso pasa a menudo. \\
La documentación es muy importante, el cliente paga por documentos de calidad. Es tan importante como el desarrollo. \\
\hline Fortalecer la programación, ya que más del $90 \%$ inicialmente se dedica a programación. \\
Debe haber mayor I+D en la carrera, debe obligarse a usar tecnologías más recientes. La contratación inicial no es por \\
el título, sino por lo que sabes hacer. Esto tanto de parte de los estudiantes y los profesores. Por ejemplo, desarrollo \\
para la Web, debe ser más actualizado. \\
Destacar que en el mundo laboral no te puedes equivocar reiteradamente. \\
También destacar que la experticia se adquiere en el trabajando. \\
\hline Fortalecer los aspectos técnicos asociados con el desarrollo de software. \\
Es importante mantener el desarrollo de proyectos poco definidos. \\
Fortalecer los requerimientos de entrada al curso. La formación previa debe ser fortalecida, se debe llegar a aplicar \\
directamente. \\
El trabajo en equipo es muy importante. Las múltiples perspectivas de las soluciones se dan allí. Así mismo, se produce \\
la delegación de responsabilidades y tiempo acotado para cada integrante. En estos espacios las soluciones son más \\
robustas.
\end{tabular}


Tabla 9 (continuación)

Se debe mantener y fortalecer el desarrollar soluciones a problemas del mundo real. Esto ayuda mucho a enfrentar el mundo real.

El trabajo debe ser en equipos. El debate de ideas y soluciones es importante en el mundo real.

Sin embargo lo anterior, no perder el trabajo individual, porque el aprendizaje esforzado individualmente igual sirve mucho.

Es importante destacar que la mayor parte del esfuerzo se va en programación. Por tanto la división del trabajo debe considerar la programación. Cada uno debe hacerse cargo proporcionalmente de una parte de esta actividad.

\section{Opinión del Experto}

Según su experiencia en la gestión de proyectos de desarrollo de software (más de 20 años) y el haber sido empleador de más de 50 exalumnos de la carrera, opinó que: i) Los procesos tanto para la ingeniería del producto como para el proyecto son claves; ii) El trabajo en equipo y multidisciplinario es una necesidad; iii) Debe fortalecerse el rol del Jefe de Proyecto; iv) Los problemas deben ser complejos. Por ejemplo, la arquitectura, en el desarrollo de software es relevante y no es fácil, aquí puede haber un tiempo extenso que el estudiante deba invertir en el proyecto. Y esta debe ser definida por el problema o el cliente; v) Las herramientas de desarrollo deben ser las que el mercado laboral está aplicando. Por ejemplo, el framework Angular JS; vi) Es muy importante la relación cliente y desarrollador; y vii) El desarrollo ágil está de moda, por lo cual debe ser más libre la elección de la metodología de desarrollo.

En resumen, los resultados ponen de manifiesto la importancia de desarrollar y estimular el trabajo de los estudiantes en proyectos reales para empresa reales (Pymes). Tanto los ExABP y ExNABP consideraron que la iniciativa (sus características y el tipo de proyecto) contribuyen significativamente en el ejercicio temprano de su profesión.

\section{CONCLUSIONES}

Se estableció una nueva estrategia pedagógica que acerca al estudiante hacia problemas reales en contextos reales. Es decir, el desarrollo de proyectos que resuelven problemas de micro empresas, trayendo al aula la realidad a la cual se verán enfrentados en el medio laboral o industrial, mejorando su aprendizaje. Esto se vio corroborado por las opiniones del experto y los exalumnos.

Posteriormente, se evaluó el impacto del uso de esta estrategia, basada en el ABP (contextualizados en problemas reales de empresas), en el ejercicio temprano de la profesión del Ingeniero Informático de la UTA. Los resultados indican que tanto la estrategia como sus características han aportado positivamente en este sentido, lo que sugiere mantenerla y potenciarla según los actores consultados.

Las entrevistas a los ExABP ya encuestados y al experto, no solo confirmaron lo indicado anteriormente, sino que además, aportaron observaciones, basadas en su ejercicio de la profesión, consideradas valiosas y concretas para mejorar la experiencia. Así, los resultados obtenidos son una contribución a la formación de Ingenieros de Software, ya que aportan con el cierre de la brecha entre las actividades teóricas y las actividades reales del desarrollo de software.

En la actualidad, durante el segundo semestre del 2017, los resultados de esta experiencia formativa, están siendo considerados para introducir cambios en el curso "Taller de Proyectos de Software" y en parte del currículo del Ingeniero Civil en Computación e Informática que se forma en la Universidad de Tarapacá, Arica, Chile.

\section{AGRADECIMIENTOS}

Este trabajo cuenta con el financiamiento del programa de Investigación en Educación de la Universidad de Tarapacá, Arica, Chile (2016-2017), en el contexto del proyecto "Cómo impactan las características de los proyectos reales desarrollados para pymes en el ejercicio profesional temprano de los estudiantes de Ingeniería Civil en Computación e Informática de la UTA" (código 8722-16). Asímismo, a los graduados y empresarios por su valiosa colaboración, interés y disposición. A todos y cada uno de ellos muchas gracias porque sin su colaboración esta investigación no podría haber concluido.

\section{REFERENCIAS}

Alsmadi, I., Abul-Huda, B., Improving Understandability in Teaching of Software Engineering and Connectivity with the Industry. IEEE Global Engineering Education Conference (EDUCON) - Learning Environments and Ecosystems in Engineering Education, 22-25, Amman, Jordan, 4-6 April (2010) 
Bará, J., Aprendizaje basado en problemas/proyectos: ¿Qué, por qué, cómo?, Institut de Ciencies de l"Educació (2003)

Benítez, A., García, M., Un Primer Acercamiento al Docente frente a una Metodología Basada en Proyectos, Formación Universitaria, 6(1), 21-28 (2013)

Bracken, B., Progressing from student to professional: the importance and challenges of teaching software engineering, Journal of Computing Sciences in Colleges, 19(2), 358-368 (2003)

Cabre, T. P., Cairol, M. T., Calafell, D. F., Ribes, M. T., Roca, J. P., Project-Based Learning Example: Controlling an Educational Robotic Arm with Computer Vision, IEEE Revista Iberoamericana de Tecnologias del Aprendizaje, 8(3), 135$142(2013)$

Cocota, J.A.N., D'Angelo, T., de Barros Monteiro, P.M., A Project-Based Learning Experience in the Teaching of Robotics. IEEE Revista Iberoamericana de Tecnologias del Aprendizaje, 10(4), 302-309 (2015)

Davies, J., De Graaff, E., Kolmos, A., PBL across the disciplines: Research into best practice: Aalborg Universitetsforlag, Aalborg, Denmark (2011)

De Graaff, E., Kolmos, A. Management of Change; Implementation of Problem-Based and Project-Based Learning in Engineering. Sense Publishers, Rotterdam, Netherlands (2007)

Fernández, F. H., Duarte, J. E., El aprendizaje basado en problemas como estrategia para el desarrollo de competencias específicas en estudiantes de Ingeniería, Formación Universitaria, 6(5), 29-38 (2013)

Galeana De La O., L. Aprendizaje Basado en Proyectos, Investigación en Educación a Distancia, goo.gl/wzvMrG, Revista electrónica Ceupromed, 1, 27 (2006)

Gallardo, C, Martínez, P., Sánchez, J., Project-based Learning Experience on Data Structures Course, IEEE Global Engineering Education Conference (EDUCON) - Learning Environments and Ecosystems in Engineering Education, 561-566, Amman, Jordan, 4-6 April (2010)

Hongzhi Song, Guodong Si, Lei Yang, Huakun Liang, Lixia Zhang, International Joint Conference of IEEE TrustCom11/IEEE ICESS-11/FCST-11 Using Project-Based Learning and Collaborative Learning in Software Engineering Talent Cultivation (2011)

Indiramma, M., Project based learning - Theoretical Foundation of Computation course, IEEE International Conference in Interactive Collaborative Learning (ICL), 841-844, Dubai, UAE, 03-06 December (2014)

Jenkins, H., Lackey, L.W., Preparing Engineering Students for Working in Teams Through Senior Design Projects, Proceedings IEEE International Professional Communication Conference, 129-136, Limerick, Ireland, 10-13 July (2005)

Kellett, C., A Project-Based Learning Approach to Programmable Logic Design and Computer Architecture, IEEE Transactions on Education, 55(3), 378-383 (2012)

Kokosy, A., Micea, M. V., Saey, P., A professional project based learning method in mobile robotics. IEEE Frontiers in Education Conference (FIE), 1-7. Madrid, Spain, October (2014)

Kolmos, A., De Graaff, E., Du, X., Diversity of PBL-PBL learning principles and models. Research on PBL practice in engineering education, Sense Publishers, 9-21, Rotterdam, Netherlands (2009)

Liu, J., Marsaglia, J., Olson, D., Teaching software engineering to make students ready to real world, Journal of Computing Sciences in Colleges, 17(6), 43-50 (2002)

Macías-Guarasa, J., Montero, J.M., San-Segundo, R., Araujo,A., Nieto-Taladriz, O., A Project-Based Learning Approach to Design Electronic Systems Curricula, IEEE Trans. on Education, 49(3), 389-397 (2006)

Martín, J. G., López, C. L., Martínez, J.E.P., Supporting the design and development of Project Based Learning courses. IEEE Frontiers in Education Conference (FIE), 1-6. Madrid, Spain, October (2014)

Martínez, F., Herrero, L., de Pablo, S., Project-Based Learning and Rubrics in the Teaching of Power Supplies and Photovoltaic Electricity, IEEE Transactions on Education, 54(1), 87-96 (2011)

Menéndez, J.M., Aprendizaje por proyectos: la experiencia en la Universidad de Castilla-La Mancha, Actas del I Encuentro Int. de Enseñanza de la Ingeniería Civil, Castilla La Mancha, Ciudad Real, España (2003)

Pérez, A., Serrano, J., Peñarrocha, I., Perez, E., Un sistema para la evaluación del aprendizaje basado en proyectos. Actas del XVI CUIEET, Cádiz, España, Septiembre (2008)

Reeves, T., Herrington, J., Oliver, R., Authentic activities and online learning. Proceedings 25th HERDSA Annual Conference, 562-562, Perth, Western Australia, 7-10 July (2002)

Savin-Baden, M., Challenging Models and Perspectives of Problem-Based Learning. Management of Change; Implementation of Problem-Based and Project-Based Learning in Engineering. Sense Publishers, 9-29, Rotterdam, Netherlands (2007)

Savin-Baden, M., Problem-Based Learning in Higher Education: Untold Stories: Untold Stories. McGraw-Hill Education, London, UK (2000) 
Song, H., Si, G., Yang, L., Liang, H., Zhang, L., Using project-based learning and collaborative learning in software engineering talent cultivation. IEEE 10th International Conference in Trust, Security and Privacy in Computing and Communications (TrustCom), 1288-1293, Changsha, China, 16-18 November (2011)

Soska, A., Schroll-Decker, I., Mottok, J., Implementation of practical exercises in software engineering education to improve the acquirement of functional and non-functional competences: A field report about project-based learning in software engineering. IEEE International Conference in Interactive Collaborative Learning (ICL), 338-345, Dubai, UAE, 03-06 December (2014)

Stiller, E., Lebland, C., Effective software engineering pedagogy, Journal of Computing Sciences in Colleges, 18(2), 124$134(2002)$

Tippelt, R., Lindemann, H., El método de proyectos. Unión Europea, Ministerio de Educación de El Salvador, München, Berlin, 13, 1-14 (2001)

Uriarte, J. (2013). La situación de la micro y pequeña empresa en Chile. SERCOTEC. Ministerio de Economía, Fomento y Turismo, Gobierno de Chile. https://goo.gl/UEHFrh (2013)

Van Hattum-Janssen, N., Lima, R., Carvalho D., Carlos L., 4th International Symposium on Project Approaches in Engineering, University of Minho and Faculty of Exact Sciences and Technology, Pontifical Catholic University of São Paulo, PUC-SP (2012)

Vargas, M.A.A., Ríos, B.L.F., Esquer, J.E.I., Camacho, J.M., Corral, L.E.V. Impacto del aprendizaje basado en proyectos implementado en una empresa escolar de Base Tecnológica dedicada al desarrollo de Soft-ware, goo.gl/wu5KPH, Rev. Electrónica de Computación, Informática, Biomédica y Electrónica (ReCIBE), 4(4), 1-47 (2015) 
